\title{
Perfil Bioquímico Sanguíneo Hepático del Cocodrilo de Tumbes (Crocodylus acutus) Criado en Cautiverio
}

\author{
Liver Blood Chemistry Profile of the American Crocodile (Crocodylus acutus) \\ RAISED IN CAPTIVITY
}

\author{
Jhonathan Zender A. ${ }^{1}$, Olga Li E. ${ }^{1,3}$, Francisco Suárez A. ${ }^{2}$, Luis Hoyos S. ${ }^{1}$, Walter \\ Silva S. ${ }^{1}$, Gianfranco Arroyo H. ${ }^{1}$, Manuel Barrios-Arpi ${ }^{1}$
}

\section{Resumen}

Se determinó el perfil bioquímico hepático del cocodrilo de Tumbes (Crocodylus acutus) criado en cautiverio en el norte del Perú. Se trabajó con 60 cocodrilos de una población de 255 animales del Zoocriadero del Centro de Acuicultura La Tuna Carranza, del Fondo Nacional de Desarrollo Pesquero (FONDEPES), ubicado en la región de Tumbes, Perú. De estos, 24 eran juveniles, 27 subadultos y 9 adultos. Se extrajo $6 \mathrm{ml}$ de sangre entera sin anticoagulante por punción del seno venoso post-occipital manipulando al animal mediante contención física. Se separó el suero mediante centrifugación y las enzimas se analizaron mediante espectrofotometría. Las variables en estudio fueron alanina aminotransferasa (ALT), aspartato amino transferasa (AST), fosfatasa alcalina (FA), bilirrubina total, bilirrubina directa, bilirrubina indirecta, proteínas totales y albúmina. Se encontró diferencia estadística $(\mathrm{p}<0.05)$ en relación a los grupos etarios con respecto a la ALT, FA, bilirrubina directa y proteínas totales. Todos los valores con excepción de la albúmina tuvieron marcadas diferencias biológicas con respecto a valores publicados por el International Species Information System.

Palabras clave: espectrofotometría, FONDEPES, Crocodylus acutus

\section{Abstract}

The liver blood chemical profile of the American crocodile (Crocodylus acutus) raised in captivity in the northern region of Peru was determined. Sixty crocodiles were sampled from a population of 255 animals belonging to the Zoocriadero del Centro de Acuicultura La Tuna Carranza, part of the National Fisheries Development Fund (FONDEPES) located in the region of Tumbes, Peru. The animals were classified as

\footnotetext{
${ }^{1}$ Laboratorio de Patología Clínica y Biología Molecular, ${ }^{2}$ Laboratorio de Medicina Veterinaria Preventiva, Facultad de Medicina Veterinaria, Universidad Nacional Mayor de San Marcos, Lima, Perú

${ }^{3}$ E-mail:olgalie@hotmail.com
} 
young $(\mathrm{n}=24)$, subadults $(\mathrm{n}=27)$ and adults $(\mathrm{n}=9)$. A blood sample $(6 \mathrm{ml})$ was taken from each animal by puncturing the post-occipital venous sinus while animals were under physical restraint. Serum was separated by centrifugation and concentration of each enzyme was measured by spectrophotometry. Variables studied were alanine aminotransferase (ALT), aspartate amino transferase (AST), alkaline phosphatase (ALP), total bilirubin, direct bilirubin, indirect bilirubin, total protein and albumin. Statistical difference $(\mathrm{p}<0.05)$ was found between age groups in ALT, FA, direct bilirubin, and total protein. All values with the exception of albumin showed high biological differences with those reported by the International Species Information System.

Key words: spectrophotometry, FONDEPES, Crocodylus acutus

\section{INTRODUCCIÓN}

El cocodrilo de Tumbes o cocodrilo americano (Crocodylus acutus) habita en el norte del Perú, desde el río Chira hasta el río Zarumilla, dentro de los esteros y manglares de Tumbes. Es un animal de elegancia, gran fortaleza e importante en la regulación del ecosistema, siendo depredador natural por excelencia, donde el animal adulto ocupa la cúspide de la pirámide alimenticia de la zona; además de cumplir un rol importante como diseminador secundario de semillas (Casas y Barrios, 2003; Escobedo y Mejía, 2003; Carvajal et al., 2005).

Es una fuente económica de importancia para el habitante de la zona, pero no se ha sabido aprovechar de manera sostenible y, por el contrario, se le caza en forma indiscriminada por su grasa, carne, dientes y, principalmente, por su piel, muy valorizada en el mercado. Actualmente se le considera como especie en peligro de extinción, listada en el Apéndice I según la Convención sobre el Comercio Internacional de Especies Amenazadas de Fauna y Flora Silvestre (CITES, 2003) y considerado en el Perú en «peligro crítico», según Decreto Supremo N. ${ }^{\circ} 034-$ 2004-AG (Escobedo, 2004; Carvajal et al., 2005, 2009).
Una de las medidas que se han tomado para la conservación de la especie es su crianza en zoológicos y la implementación de un zoocriadero en Tumbes con fines reproductivos y con miras en el largo plazo a la reintroducción de especímenes al medio ambiente (FONDEPES, 2006).

La evaluación hematológica y bioquímica incluye una gama de métodos sencillos que permiten obtener excelentes indicadores del estado de salud de un individuo, así como de su estado reproductivo; además, es una herramienta importante en varios procedimientos médicos relacionados a la especie, ya sean profilácticos, rutinarios o curativos (Momtilla et al., 2006).

No existe información sobre el perfil bioquímico hepático de Crocodylus acutus hechos en el Perú. A nivel internacional, solo se encontró reportes sobre valores de bioquímica hepática para la especie publicados por International Species Information System (2002), los cuales están basados en una cantidad pequeña y variable de animales, sin especificar edad, sexo, manejo, época del año, localización geográfica o estado reproductivo.

Este trabajo, al ser pionero en búsqueda de parámetros bioquímicos del C. acutus busca incentivar futuras investigaciones que puedan complementar los resultados hallados. 


\section{Materiales y Métodos}

Se trabajó con la población de cocodrilos (Crocodylus acutus) criados en cautiverio $(n=255)$ en el Zoocriadero del Centro de Acuicultura La Tuna Carranza, del Fondo Nacional de Desarrollo Pesquero (FONDEPES), ubicado en Puerto Pizarro, departamento de Tumbes, Perú. Se dividió la población siguiendo la clasificación de Thorbjarnarson (1989) en juveniles $(<90 \mathrm{~cm})$, subadultos $(90-180 \mathrm{~cm})$ y adultos $(>180 \mathrm{~cm})$, y se utilizó una muestra de 60 animales, siendo 24 juveniles, 27 sub-adultos y 9 adultos, según la fórmula de estratificación (Da Silva, 2004).

Los cocodrilos estaban clínicamente sanos y fueron criados en las mismas condiciones de alojamiento, alimentación, manejo y control sanitario. La alimentación se suministraba por las mañanas y básicamente con- sistía en hígado, pollo y pescado, suplementado con sales minerales y vitaminas. El suministro de la ración alimenticia era de tres veces por semana para los cocodrilos juveniles, mientras que para los sub-adultos y adultos era entre dos y una vez por semana.

La extracción de muestras de sangre se llevó a cabo durante el invierno, en horas de la mañana y con los animales en ayuno. Se minimizó en lo posible la manipulación del animal y el tiempo en colectar la muestra para evitar el estrés del animal. Las muestras fueron recolectadas según la técnica de Jacobson (1993) por punción del seno venoso postoccipital (Fig. 1), empleando agujas de $21 \mathrm{G}$ y jeringas de $10 \mathrm{ml}$. Se colectó $6 \mathrm{ml}$ de sangre, aproximadamente, y la sangre se trasvasó a tubos sin anticoagulante. La temperatura ambiental promedio durante los tres días del muestreo estuvo entre 25.3 a $26.1{ }^{\circ} \mathrm{C}$, y la humedad relativa entre 89.6 y $91.5 \%$ (SENAMHI, 2009).

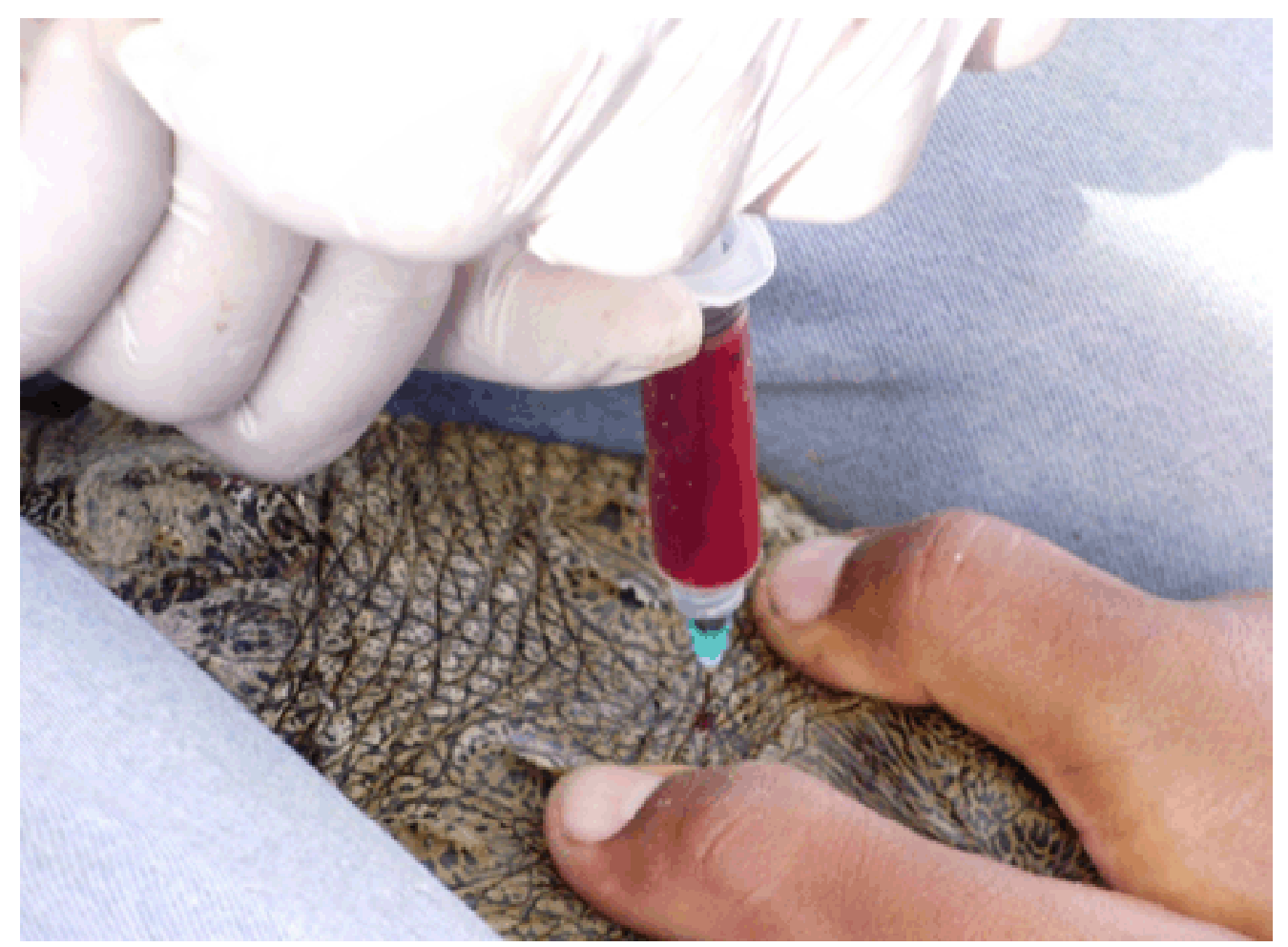

Figura 1. Toma de muestra de sangre del seno venoso post-occipital en el cocodrilo de Tumbes 
Las muestras fueron llevadas al laboratorio del Centro de Investigación de Cisticercosis de Tumbes para la obtención del suero por centrifugación a $3500 \mathrm{rpm}$ por $10 \mathrm{minu}-$ tos. Las muestras de suero fueron trasladadas bajo refrigeración al Laboratorio de Patología Clínica de la Facultad de Medicina Veterinaria de la Universidad Nacional Mayor de San Marcos, Lima. Se determinaron los siguientes parámetros bioquímicos por espectrofotometría según las especificaciones del fabricante (Wiener Lab): alanina aminotransferasa (ALT) y aspartato aminotransferasa (AST) mediante método cinético optimizado con longitud de onda de $340 \mathrm{~nm}$, fosfatasa alcalina (FA) mediante método cinético optimizado con longitud de onda de $405 \mathrm{~nm}$, bilirrubina (BT, BD, BI) y proteínas totales mediante método colorimétrico con longitud de onda de $540 \mathrm{~nm}$, y albúmina mediante método colorimétrico con longitud de onda de $625 \mathrm{~nm}$. Se empleó el espectrofotómetro Riele 5010 V5+ (Berlín, Alemania).

Se hizo estadística descriptiva, utilizando la media aritmética como medida de tendencia central y la desviación estándar como medida de dispersión. Cada una de las variables fue sometida a un test de normalidad para verificar que los valores seguían la distribución normal, y aquellos que no se distribuían de forma normal fueron transformados para poder realizar una prueba paramétrica (análisis de varianza - ANOVA) y determinar diferencias entre grupos etarios; sin embargo, aquellas que no se pudieron transformar a una distribución normal fueron tratadas con pruebas no paramétricas (Kruskal-Wallis).

Variables con significancia estadística en el ANOVA fueron sometidas a la prueba de Tukey y a las analizadas con Kruskal-Wallis se les aplicó un análisis de varianzas por rangos. Todos los análisis estadísticas se hicieron utilizando el programa estadístico STATA.

\section{Resultados y Discusión}

Los valores de bioquímica hepática para el cocodrilo de Tumbes se muestran en el Cuadro 1, en tanto que en el Cuadro 2 se puede observar los valores según el grupo etario.

Las diferencias estadísticas en ALT entre juveniles y adultos $(\mathrm{p}<0.05)$ podrían deberse a factores intrínsecos del animal tanto

Cuadro 1. Valores de bioquímica hepática en 60 cocodrilos de Tumbes (Crocodylus acutus) de un zoocriadero del norte del Perú

\begin{tabular}{lcccc}
\hline Parámetro $^{1}$ & Media & d.e. & $\begin{array}{c}\text { Valor } \\
\text { mínimo }\end{array}$ & $\begin{array}{c}\text { Valor } \\
\text { máximo }\end{array}$ \\
\hline ALT (UI/L) & 25.65 & 10.84 & 6 & 59 \\
AST (UI/L) & 30.25 & 17.96 & 10 & 150 \\
FA (UI/L) & 37.33 & 20.19 & 6 & 88 \\
Bilirrubina total (mg/dL) & 3.54 & 4.52 & 0.52 & 28.32 \\
Bilirrubina directa (mg/dL) & 1.41 & 1.67 & 0.26 & 7.73 \\
Bilirrubina indirecta (mg/dL) & 2.13 & 3.71 & 0.25 & 25.74 \\
Proteínas totales (g/dl) & 4.62 & 1.14 & 2.8 & 8 \\
Albúmina (g/dl) & 1.9 & 0.41 & 0.9 & 2.9 \\
\hline
\end{tabular}

${ }^{1}$ ALT: alanina aminotransferasa; AST: aspartato aminotransferasa; FA: fosfatasa alcalina 
Cuadro 2. Valores comparativos de bioquímica hepática en cocodrilos de Tumbes (Crocodylus acutus) juveniles $(\mathrm{n}=24)$, sub-adultos $(\mathrm{n}=27)$ y adultos $(\mathrm{n}=9)$

\begin{tabular}{lccc}
\hline Parámetro $^{1}$ & $\begin{array}{c}\text { Juveniles } \\
\text { media } \pm \text { e.e. }\end{array}$ & $\begin{array}{c}\text { Sub-Adultos } \\
\text { media } \pm \text { e.e. }\end{array}$ & $\begin{array}{c}\text { Adultos } \\
\text { media } \pm \text { e.e. }\end{array}$ \\
\hline ALT (UI/L) & $30.75 \pm 2.68^{\mathrm{a}}$ & $22.41 \pm 1.61^{\mathrm{b}}$ & $21.78 \pm 1.36^{\mathrm{b}}$ \\
AST (UI/L) & $36.08 \pm 5.43^{\mathrm{a}}$ & $25.89 \pm 1.31^{\mathrm{a}}$ & $27.78 \pm 1.45^{\mathrm{a}}$ \\
FA (UI/L) & $43.42 \pm 4.56^{\mathrm{a}}$ & $37.70 \pm 3.49^{\mathrm{b}}$ & $20 \pm 2.33^{\mathrm{a} b}$ \\
Bilirrubina total $(\mathrm{mg} / \mathrm{dL})$ & $4.56 \pm 0.84^{\mathrm{a}}$ & $2.30 \pm 0.34^{\mathrm{a}}$ & $4.55 \pm 3.01^{\mathrm{a}}$ \\
Bilirrubina directa (mg/dL) & $2.21 \pm 0.46^{\mathrm{a}}$ & $0.87 \pm 0.15^{\mathrm{a}}$ & $0.92 \pm 0.29^{\mathrm{a}}$ \\
Bilirrubina indirecta (mg/dL) & $2.35 \pm 0.57^{\mathrm{a}}$ & $1.43 \pm 0.24^{\mathrm{a}}$ & $3.63 \pm 2.78^{\mathrm{a}}$ \\
Proteínas totales $(\mathrm{g} / \mathrm{dl})$ & $3.99 \pm 0.1^{\mathrm{a}}$ & $4.89 \pm 0.25^{\mathrm{a}}$ & $5.5 \pm 0.31^{\mathrm{a}}$ \\
Albúminas $(\mathrm{g} / \mathrm{dl})$ & $1.84 \pm 0.06^{\mathrm{a}}$ & $1.93 \pm 0.1^{\mathrm{a}}$ & $1.96 \pm 0.13^{\mathrm{a}}$ \\
\hline
\end{tabular}

${ }^{a, b}$ Superíndices diferentes dentro de filas indican diferencia estadística $(p \varangle 0.05)$

${ }^{1}$ ALT: alanina aminotransferasa; AST: aspartato aminotransferasa; FA: fosfatasa alcalina

como a factores extrínsecos como el método de captura, estado de salud y fisiológico de los animales al momento del muestreo, alojamiento de los animales y su alimentación (Rojas et al., 2007).

Las marcadas diferencias en FA entre los grupos etarios $(\mathrm{p}<0.05)$ muestran una proporción inversa entre la edad del animal y la cantidad de enzima; lo cual concuerda con Coppo et al. (2005), quienes encontraron en individuos de la familia Crocodylidae que a mayor edad se encontraba menor cantidad de FA en suero y lo relacionaron con la disminución gradual del desarrollo óseo.

Los niveles más elevados de bilirrubina directa se encontraron en los juveniles ( $\mathrm{p}<0.05)$. Coppo et al. (2005) compararon en caimanes el perfil hepático entre estaciones del año y encontraron que en el verano se presentaban los valores más elevados debido al mayor consumo de alimentos y aumento del metabolismo. De ser así, en el caso de la mayor cantidad de bilirrubina en juveniles podría deberse al metabolismo más eficiente propio de su edad, y a que recibían una alimentación más frecuente (3 veces por semana versus 1-2 veces por semana en los animales de mayor edad).

Los valores de proteína total aumentaron significativamente con la edad $(\mathrm{p}<0.05)$, aunque los valores de albúmina se mantuvieron constantes entre los grupos etarios. La explicación podría sustentarse en el trabajo de Coppo et al. (2006) con el desarrollo ontogénico de un anfibio donde explica que este aumento puede deberse a la mayor cantidad de anticuerpos debido a la maduración progresiva del sistema inmunológico.

Los valores de AST, bilirrubina total e indirecta no fueron afectados por la edad de los animales.

El Cuadro 3 enfrenta los valores encontrados en el presente estudio con valores publicados por otros autores. Se encontró que los valores obtenidos en este estudio difieren sustancialmente de los valores publicados por ISIS (2002) en cuanto a ALT, AST, FA, bilirrubina total directa e indirecta, y proteínas totales, aunque similares a los valores de albúmina. Esta variabilidad podría atribuirse a una serie de factores tales como lugar de 
Cuadro 3. Valores comparativos de bioquímica hepática en el cocodrilo de Tumbes (Crocodylus acutus)

\begin{tabular}{lccccc}
\hline & $\begin{array}{c}\text { Crocodylus } \\
\text { acutus }\end{array}$ & $\begin{array}{c}\text { Crocodylus } \\
\text { acutus }\end{array}$ & $\begin{array}{c}\text { Caiman } \\
\text { crocodylus }\end{array}$ & $\begin{array}{c}\text { Caiman } \\
\text { latirostris }\end{array}$ & $\begin{array}{c}\text { Caiman } \\
\text { yacare }\end{array}$ \\
\cline { 2 - 6 } & $\begin{array}{c}\text { Zender } \text { et } \\
\text { al. }(2016)^{1}\end{array}$ & $\begin{array}{c}\text { ISIS } \\
(2002)\end{array}$ & $\begin{array}{c}\text { Rojas } \text { et al. } \\
(2007)\end{array}$ & $\begin{array}{c}\text { Coppo } \text { et al. } \\
\text { (2005) }\end{array}$ \\
\hline ALT (UI/L) & $25.7 \pm 10.8$ & $74 \pm 93$ & $27.2 \pm 8.8$ & $14.4 \pm 3$ & $15.1 \pm 3.2$ \\
AST (UI/L) & $30.3 \pm 18.0$ & $78 \pm 64$ & $56.1 \pm 18.2$ & $56.9 \pm 9.8$ & $72.4 \pm 12.6$ \\
FA (UI/L) & $37.3 \pm 20.2$ & $67 \pm 72$ & & $54.2 \pm 7.2$ & $55.8 \pm 7.6$ \\
$\begin{array}{l}\text { Bilirrubina total } \\
\text { (mg/dL) }\end{array}$ & $3.5 \pm 4.5$ & $0.1 \pm 0.1$ & & & \\
$\begin{array}{l}\text { Bilirrubina directa } \\
\text { (mg/dL) }\end{array}$ & $1.4 \pm 1.7$ & $0.1 \pm 0.1$ & & & \\
$\begin{array}{l}\text { Bilirrubina indirecta } \\
\text { (mg/dL) }\end{array}$ & $2.1 \pm 3.7$ & $0 \pm 0$ & & & \\
$\begin{array}{l}\text { Proteínas totales } \\
\text { (g/dl) }\end{array}$ & $4.6 \pm 1.1$ & $5.4 \pm 1.4$ & $4.8 \pm 1.1$ & & \\
Albúminas (g/dl) & $1.9 \pm 0.4$ & $1.8 \pm 0.3$ & $0.5 \pm 0.1$ & & \\
\hline
\end{tabular}

${ }^{1}$ Presente estudio

venopunción, tiempo de extracción de la sangre, falta de ayuno previo, edad, sexo, época del año, tamaño de muestra, retraso en procesos analíticos y técnicas usadas en el procesamiento de la muestra (Coppo et al., 2005).

Cabe resaltar que las muestras de ISIS no se considerarían representativas a la población debido a que se tratan de pequeñas cantidades (menos de 10 individuos) que varían por cada prueba; además, no todos esos animales necesariamente provienen del mismo criadero.

\section{Conclusiones}

- Se estableció valores referenciales de ALT, AST, FA, bilirrubina, proteínas totales y albúminas séricas para el cocodrilo de Tumbes, criado en cautiverio en el Zoocriadero del Centro de Acuicultura
La Tuna Carranza, en la ciudad de Tumbes, Perú.

- Se encontraron diferencias biológicas importantes entre los valores obtenidos en este estudio y los reportados por ISIS, en todos los parámetros hepáticos con excepción de la albúmina.

- Se encontró diferencia estadística significativa en relación a los grupos etarios con respecto a la ALT, FA, bilirrubina directa y proteínas totales.

\section{Agradecimientos}

Al personal técnico del Laboratorio de Patología Clínica y a los miembros del laboratorio del Centro de Investigación de Cisticercosis de Tumbes, Dres. Guillermo Gonzales, Viterbo Ayvar, André Díaz, Abiud Noriega, Alan García y Heidy Nizama por su invaluable apoyo en el desarrollo de la investigación. 


\section{Literatura Citada}

1. Carvajal R, Saavedra M, Alava J. 2005. Ecología poblacional, distribución y estudio de hábitat de Crocodylus acutus (Cuvier, 1807) en la «Reserva de producción de fauna manglares El Salado» del estuario del Golfo de Guayaquil, Ecuador. Rev Biol Marina Oceanografía 40: 141-150.

2. Carvajal R, Saavedra M, Morales M. 2009. Cocodrilo americano o de la costa. DarwinNet [Internet]. Disponible en: h t t p : / / www.darwinnet.org/ index.php?option=com_content $\&$ view= article \&id $=842 \% 3$ Acocodrilo-americano-o-de- 1 a - costa \& catid $=60 \% 3$ Aespecie-del-mes\&Itemid=1

3. Casas G Barrios G 2003. Hábitos alimenticios de Crocodylus acutus (Reptilia: Crocodylidae) determinado por el análisis de sus excretas en la costa de Jalisco, México. Anales Inst Biol Univ Nac Autón Méx, Ser Zool 74: 35-42.

4. [CITES] Convenio Internacional para el Tráfico de Especies Amenazadas de Flora y Fauna. 2003. [Internet]. Disponible en: http://www.cites.org/esp/app/ appendices.shtml

5. Coppo J, Mussart N, Barboza N, Fioranelli S, Koza G, Prado W. 2006. Physiological changes in serum glucidic and nitrogenic analytes from captive Argentine authoctonous caimans. Rev Vet 17: 103-108.

6. Coppo N, Coppo J, Barboza N, Prado W. 2005. Serum enzymatic activities in captive northeastern-Argentina caymen (Crocodylia: Crocodylidae). Rev Vet 16: 16-20.

7. Da Silva N. 2004. Amostragem probabilística. $2^{\circ}$ ed. Sao Paulo: USP. 235 p.

8. Escobedo A. 2004. Avances en el conocimiento y el estado actual de conservación del Cocodrilo de Tumbes (Crocodylus acutus Cuvier, 1807). Rev
Perú Biol 11: 203-208. doi: 10.15381/ rpb.v11i2.2457

9. Escobedo A, Mejía F. 2003. El «cocodrilo de Tumbes» (Crocodylus acutus Cuvier 1807): estudio preliminar de su estado actual en el norte del Perú. Ecol Aplicada 2(1): 133-135.

10. [FONDEPES] Fondo Nacional de Desarrollo Pesquero. 2006. Manejo en cautiverio del cocodrilo americano Crocodylus acutus. Lima: FONDEPES. Documento técnico. $34 \mathrm{p}$.

11. [ISIS] International Species Information System. 2002. Reference ranges for physiological data values of American crocodile (Crocodylus acutus), USA. [Internet]. Disponible en: http://www.isis.org.com

12. Jacobson E. 1993. Blood collection techniques in reptiles: Laboratory investigations. In: Fowler ME (ed). Zoo and wild animal medicine. Current therapy 3. Philadelphia: WB Saunders. p 144-146.

13. Montilla A, Hernández J, Alvarado M. 2006. Valores hematológicos en la tortuga verde (Chelonia mydas) presente en la alta Guajira. Rev Cient FCV-LUZ 16:219-226.

14. Rojas G, Ramírez J, Jibaja M. 2007. Estudio de bioquímica sérica del caimán blanco (Caiman crocodylus) mantenidos en cautiverio en el parque zoológico Huachipa Lima-Perú. En: I Congreso de Fauna Silvestre de la Ciudad Autónoma de Buenos Aires. Argentina.

15. [SENAMHI] Servicio Nacional de Meteorología e Hidrología del Perú. 2009. [Internet]. Disponible en: http:// www.senamhi.gob.pe/

16. Thorbjarnarson J. 1989. Ecology of the American crocodile, Crocodylus acutus. In: Crocodiles, their ecology, management, and conservation. Switzerland: IUCN Publications New Series. p 229-259. 